\title{
La teoría de la objetivación. Una perspectiva vigotskiana sobre conocer y devenir en la enseñanza y el aprendizaje de las matemáticas, de Luis Radford
}

\author{
Armando Solares-Rojas ${ }^{1}$
}

Luis Radford nos ofrece en este libro un recuento sistemático de la Teoría de la Objetivación (TO). Ubica a la TO como una teoría sociocultural inspirada en el materialismo dialéctico contemporáneo; es decir, como una teoría que concibe al aprendizaje como un proceso histórico-cultural colectivo. Por "colectivo" la TO no se refiere a un conjunto de individuos que negocian idiosincráticamente los significados de sus conocimientos matemáticos; en la TO el colectivo se refiere a individuos que trabajan juntos para satisfacer las necesidades comunitarias del grupo; colectivo se refiere a maestros y estudiantes trabajando y aprendiendo juntos, co-produciendose a sí mismos en un transfondo histórico y cultural específico. En ese sentido, una característica fundamental de la TO es dicha labor conjunta.

La TO desconstruye y replantea nuestros conceptos de maestro y alumno. Va más allá del concepto del maestro como "patriarca del conocimiento", como poseedor, validador y gestor del conocimiento matemático que ayuda a los estudiantes a comprender los contenidos curriculares. La TO entiende al maestro y los estudiantes como seres en permanente flujo y cambio, que se co-producen a sí mismos mediante su labor educativa conjunta, con la cual se comprometen

${ }^{1}$ Departamento de Matemática Educativa, Cinvestav, asolares@cinvestav.mx, orcid.org/00000002-3478-1736 
éticamente y en la cual sufren y luchan juntos, pero también encuentran disfrute y realización.

Inspirada en las filosofías de Hegel y Marx, la obra de Vygotsky y el concepto de educación de Freire, la TO se plantea el siguiente doble objetivo:

- ofrecer una concepción teórica del aprendizaje como un genuino proceso histórico-cultural colectivo, y

- explorar las condiciones pedagógicas concretas y específicas que hacen posible un aprendizaje colectivo genuino.

En concordancia con el principio dialéctico del materialismo de que el proceso de conocer va/asciende de lo abstracto a lo concreto, Radford comienza el primero de los diez capítulos del libro abordado la cuestión general de lo que es una teoría en educación matemática. A lo largo del libro, nos proporciona tanto elementos teóricos abstractos como sus concreciones a través de ejemplos provenientes tanto de la historia de las matemáticas como de situaciones concretas de aula. En el primer capítulo, Radford plantea que una teoría en educación matemática puede considerarse como un sistema que siempre está cambiando y que está constituido de componentes interrelacionados. Discute las diferencias de la aproximación de la TO con otras teorías, en particular, las constructivistas y la Teoría de las Situaciones Didácticas. En este capítulo Radford presenta el objetivo de la TO y lo enmarca en una concepción de la educación matemática "como un esfuerzo político, social, histórico y cultural dirigido a la creación dialéctica de sujetos reflexivos y éticos que se posicionan críticamente en discursos y prácticas matemáticas histórica y culturalmente constituidos, y que ponderan nuevas posibilidades de acción y pensamiento" (Radford, 2021, pp. 15-16).

El capítulo 2 se dedica a una descripción general de la TO en el marco de las teorías socioculturales en educación matemática. El autor nos ofrece una discusión de algunos de los elementos básicos de la TO, como los conceptos de aprendizaje, maestro, estudiantes, artefactos, signos, lenguaje y corporeidad (embodiment). Con estos elementos, Radford nos propone una primera descripción de la TO como una manera de comprender la enseñanza y el aprendizaje no como el resultado de los propios actos de los individuos, sino como procesos de conocer (knowing) y devenir (becoming) cultural e históricamente situados.

La cuestión fundamental del conocimiento y su aprendizaje se aborda en el capítulo 3, introduciendo los elementos teóricos saber (knowledge) y conocimiento (knowing). En la TO, la relación entre estos dos elementos es dialéctica en un 
sentido materialista. Radford nos dice "el saber (knowledge) es una entidad general, un sistema de formas o arquetipos de pensamiento, acción y reflexión constituidos histórica y culturalmente a partir del trabajo colectivo material, corporizado y sensible" (pp. 40-41); mientras que "el conocimiento (knowing) es el contenido conceptual concreto a través del cual el saber se corporiza, materializa o actualiza" (p. 51). Al final del capítulo, Radford nos ofrece una hermosa metáfora de las diferencias entre estos dos elementos teóricos a partir del análisis de dos interpretaciones distintas (dos concreciones) de la Séptima sinfonía de Beethoven, dirigidas por Karajan y por Rubinstein, respectivamente.

Con estos elementos, en los capítulos 4 y 5 se discuten a detalle los conceptos de aprendizaje y objetivación. Si bien objetivación aparece en distintas teorías, su significado en la TO proviene de la fenomenología de Hegel y se entiende como "un proceso social mediante el cual el individuo adquiere progresivamente una conciencia crítica de las formas histórico-culturales de pensar y hacer" (p. 83). Pero es fundamental señalar que durante estos procesos la conciencia - en tanto relación y auto-posicionamiento del individuo con respecto al mundo exterior- se forma y se transforma. Por su parte, "El aprendizaje consiste en percibir activa y creativamente estos sistemas histórico-culturales de pensamiento y acción" (p. 84); es decir, aprender es volverse conciente y crear conciencia crítica de estos sistemas.

El capítulo 6 presenta un panorama de lo que se entiende por corporeidad (embodiment) y su importancia en la TO; a partir de ello, se introduce el concepto de cognición sensible o sensual. En relación con la corporeidad (embodiment), Radford sostiene que el cuerpo es un lugar para la constitución de los significados matemáticos subjetivos de los estudiantes y del sistema cultural e histórico de significados matemáticos que ofrece la escuela. Por su parte, "la cognición sensible (sensuous cognition) se refiere a la idea de que la cognición humana es una forma sensible, multimodal, cultural e históricamente constituida de responder, actuar, sentir, transformar y dar sentido al mundo de manera creativa" (p. 108). Para la TO el cuerpo, los sentidos y los objetos percibibles mediante los sentidos no se consideran entidades dadas, sino son entidades mutuamente transformadas por la actividad histórico-cultural.

Como toda teoría de educación matemática, el diseño de tareas constituye un aspecto metodológico de suma importancia. Esl capítulo 7 se dedica a la metodología de configuración de las actividades de enseñanza y aprendizaje en la TO. Este capítulo provee de elementos para la elección de problemas matemáticos a presentar a los estudiantes, el orden en que es conveniente 
presentarlos, y las formas de colaboración que pueden ayudar a los estudiantes a resolver estos problemas. Radford propone que la organización de las tareas se efectúe mediante lo que llama unidad conceptual y contextual (la interrelación entre los problemas en términos de un contexto específico), con la cual se busca crear niveles profundos de conceptualización matemática mediante una curva de creciente complejidad conceptual de los problemas que se presenta a los alumnos.

El capítulo 8 se dedica a discutir una de las cuestiones más complejas pero menos atendidas de las teorías educativas socioculturales: la naturaleza cultural del pensamiento matemático. Desde mi punto de vista, se trata de uno de los capítulos más originales e interesantes de este libro. Después de discutir diferentes maneras de entender lo que son la "cultura" y las "diferencias culturales", Radford nos presenta qué se entiende por cultura desde el enfoque dialéctico materialista de la TO. A partir de estos posicionamientos, discute tres formas distintas de pensamiento matemático de la antigua Grecia, asociadas intrínsecamente a prácticas sociales que responden a las demandas de distintos momentos históricos, políticos y económicos de la sociedad griega. Más allá del pensamiento matemático teoremático frecuentemente asociado con los Elementos de Euclides, Radford nos ofrece en este capítulo un análisis profundo de las tradiciones del pensamiento matemático de los geómetras prácticos-harpēdonaptai-, dedicados a la medición de terrenos usando cuerdas, y de los calculistas con guijarros, dedicados a cálculos numéricos para negocios comerciales y finanzas de la época.

El capítulo 9 enfoca la discusión del proceso de subjetivación. Nuevamente, mediante ejemplos provenientes de la historia de las matemáticas y de situaciones de clase, el autor nos presenta procesos de subjetivación -de posicionamiento en las prácticas matemáticas específicas- entrelazados con los procesos de objetivación, a través de los cuales se da el aprendizaje.

Finalmente, el capítulo 10 nos reserva uno de los aspectos más característicos y originales de la TO, en tanto teoría de educación matemática: su ética comunitaria. Radford nos propone concebir y practicar una ética de la liberación, entendida "como una fuerza liberadora que puede ayudar a enfrentarnos a las fuentes y estructuras históricas, políticas y económicas de la opresión, la violencia y la desigualdad" (p. 206). Para Radford la TO no solo es un lente teórico a través del cual mirar e interpretar los fenómenos del aprendizaje; también es una invitación enfática y sólidamente fundamentada a transformar las prácticas pedagógicas matemáticas, a transformar el aula en un lugar donde los 
estudiantes puedan encontrar conocimientos y voces culturales matemáticas diversas, y al mismo tiempo vivir experiencias de vida colectiva, de solidaridad, de pluralidad y de inclusión.

Para terminar esta reseña, un comentario personal. Coincido profundamente con el autor cuando nos propone mirar la labor educativa conjunta como portadoras de esperanza. Esperanza en el sentido de Freire, "la esperanza de que juntos podamos hacer del mundo que heredamos y del que somos responsables, un lugar mejor: un mundo digno para quienes vengan y vivan como humanos: es decir, en paz y en solidaridad" (p. 209).

\section{REFERENCIAS}

Radford, L. (2021). The theory of objectification: A Vygotskian perspective on knowing and becoming in mathematics teaching and learning. Brill/ Sense: Leiden, The Netherlands.

ARMANDO SOLARES-ROJAS

Dirección: Departamento de Matemática Educativa, Cinvestav asolares@cinvestav.mx 